\title{
INTERPOLATORY PRODUCT INTEGRATION FOR RIEMANN-INTEGRABLE FUNCTIONS
}

\author{
PHILIP RABINOWITZ ${ }^{l}$ AND WILLIAM E. SMITH ${ }^{2}$
}

(Received 19 September 1986; revised 30 October 1986)

\begin{abstract}
Conditions are found for the convergence of interpolatory product integration rules and the corresponding companion rules for the class of Riemann-integrable functions. These conditions are used to prove convergence for several classes of rules based on sets of zeros of orthogonal polynomials possibly augmented by one or both of the endpoints of the integration interval.
\end{abstract}

\section{Introduction}

In the study of the convergence of interpolatory product integration rules, it is much easier to establish results for $C$, the space of continuous functions than for $R$, the space of Riemann-integrable functions. This is so since convergence in $C$ follows easily from results on mean convergence of Lagrange interpolation which are proved for $C$. On the other hand, the extension of these results to $R$ may require the setting up of some complicated machinery as was done by Sloan and Smith [5] and by Rabinowitz [3] who followed in their footsteps. In the present paper we shall prove a general theorem which will enable us to extend convergence results in $C$ to similar results in $R$. In addition, we shall give an additional sufficient condition for the convergence of the companion rule defined in [5]. We shall then apply this theorem to recover the results in [5] and [3] as well as to prove some new results. Unfortunately, this theorem will not enable us to extend

\footnotetext{
${ }^{1}$ Department of Applied Mathematics, Weizmann Institute of Science, Rehovot 76100, Israel.

${ }^{2}$ Department of Applied Mathematics, University of New South Wales, P. O. Box 1, Kensington, N.S.W. 2033, Australia.

( Copyright Australian Mathematical Society 1987, Serial-fee code 0334-2700/87
} 
to $R$ the convergence results in [2] in those cases not covered in [3]. However, by applying the ideas used in the proof of our theorem, we shall be able to extend all the results in [2] to the space $R$.

\section{Preliminaries}

We shall be concerned with integrals of the form

$$
I(k f)=\int_{-1}^{1} k(x) f(x) d x,
$$

where $k \in L_{1}$ and $f \in R$. We shall approximate these integrals by integration rules of the form

$$
I_{n}(f ; k)=\sum_{i=1}^{n} w_{i n}(k) f\left(x_{i n}\right), \quad n=1,2, \ldots,
$$

where the sets $X_{n}=\left\{x_{i n}: i=1, \ldots, n\right\}$ are specified in advance and the $w_{i n}(k)$ are chosen such that $I_{n}(f ; k)=I(k f)$ whenever $f \in P_{n-1}$, the space of all polynomials of degree $\leqslant n-1$. If we define the Lagrange interpolating polynomial $L_{n-1}^{f}$ by

$$
L_{n-1}^{f}(x)=\sum_{i=1}^{n} l_{i n}(x) f\left(x_{i n}\right)
$$

where

$$
l_{i n}(x)=\prod_{\substack{j=1 \\ j \neq i}}^{n} \frac{x-x_{j n}}{x_{i n}-x_{j n}}=\frac{\pi_{n}(x)}{\left(x-x_{i n}\right) \pi_{n}^{\prime}\left(x_{i n}\right)}
$$

and

$$
\pi(x)=\prod_{j=1}^{n}\left(x-x_{j n}\right),
$$

then as is well known [1, p. 75], $I_{n}(f ; k)=I\left(k L_{n-1}^{f}\right)$ and $w_{i n}(k)=I\left(k l_{i n}\right)$.

A particular choice of $k$ is the admissible weight function $w$ where $w \in A$, the set of admissible weight functions on $[-1,1]$ for which $w \in L_{1}$ and $w>0$ almost everywhere. With each $w \in A$, there is associated a sequence of orthonormal polynomials

$$
\left\{p_{n}(x ; w)=k_{n}(w) x^{n}+\cdots, k_{n}(w)>0\right\}
$$

such that $\left(p_{n}, p_{m}\right)=I\left(w p_{n} p_{m}\right)=\delta_{n m}$. If we choose $X_{n}$ to be the set of zeros of

$$
q_{n}^{(r, s)}(x)=w^{(r, s)}(x) p_{n-r-s}(x ; w),
$$


where $r, s \in\{0,1\}$ and $w^{(r, s)}(x)=(1-x)^{r}(1+x)^{s}$, and if $w / w^{(r, s)} \in A$, then rule (2) with $k=w / w^{(r, s)}$ is a Gauss rule when $r=s=0$, a Lobatto rule when $r=s=1$ and a Radau rule when $r+s=1$ and it is exact for all $f \in P_{2 n-1-r-s}$.

A particular admissible weight function which has been studied in [2] and [3] is the generalized smooth Jacobi weight function. We say that $w \in G S J$ if

$$
w(x)=\psi(x)(1-x)^{\alpha}(1+x)^{\beta} \prod_{k=1}^{m}\left|t_{k}-x\right|^{\gamma_{k}},
$$

where $-1<t_{1}<\cdots<t_{m}<1 ; \alpha, \beta, \gamma_{k}>-1, k=1, \ldots, m \geqslant 0, \psi>0, \psi \in C$ and the modulus of continuity of $\psi, \omega(t ; \psi)$ satisfies

$$
\int_{0}^{1} \omega(t ; \psi) t^{-1} d t<\infty
$$

We shall now introduce some notation from [5] for norms of functions and operators. The space $L_{q}^{(\rho)}, 1 \leqslant q \leqslant \infty$, where $\rho$ is a nonnegative measurable function that vanishes on at most a set of measure zero, is the space of measurable functions $g$ for which

$$
\|g\|_{q}^{(\rho)} \equiv\|g \rho\|_{q}<\infty .
$$

If $\rho \in L_{q}$, the norm $\left\|L_{n}\right\|_{\infty q}^{(\rho)}$ of the Lagrangian interpolation operator is given by

$$
\left\|L_{n}\right\|_{\infty q}^{(\rho)}=\sup _{f \in C} \frac{\left\|L_{n}^{f}\right\|_{q}^{(\rho)}}{\|f\|_{\infty}} .
$$

If $p^{-1}+q^{-1}=1$ and $\rho \in L_{q}$, it follows from Hölder's inequality that $L_{p}^{(1 / \rho)} \subseteq L_{1}$. We also note that the set of polynomials is dense in $L_{p}^{(1 / p)}$.

We are now ready to state and prove our theorems and corollaries.

\section{Extension to Riemann-integrable functions of convergence results for continuous functions}

THEOREM 1. Let $\left\{X_{n}\right\}$ be a sequence of point sets such that, for some $v \in A$ and every $n$, the rule $I_{n}(f ; v)$ based on $X_{n}$ is exact for all polynomials of degree $\leqslant c n$ for some $c>1$ and all sufficiently large $n$, and such that $I_{n}(f ; v) \rightarrow I(v f)$ for all $f \in R$. Assume that for some $p, 1<p<\infty$, there exists a function $\rho \in L_{q}$, $p^{-1}+q^{-1}=1$, such that $I_{n}(f ; k) \rightarrow I(k f)$ for all $f \in C$ and all $k \in L_{p}^{(1 / \rho)}$. Then $I_{n}(f ; k) \rightarrow I(k f)$ for all $f \in R$ and the same $k$. If in addition $\sum_{i=1}^{n}\left|w_{i n}(v)\right| \rightarrow I(v)$, in particular, if $w_{i n}(v)>0, i=1, \ldots, n$, for $n$ sufficiently large, then the companion rule $\rightarrow I(|k| f)$ for all $f \in R$ and the same $k$. 
Proof. As in [5], we have that for any $k^{\prime}$,

$$
\begin{aligned}
\left|I(k f)-I_{n}(f ; k)\right| \leqslant M(f)\left[I\left(\left|k-k^{\prime}\right|\right)\right. & \left.+\sum_{i=1}^{n}\left|w_{i n}\left(k-k^{\prime}\right)\right|\right] \\
& +\left|\sum_{i=1}^{n} w_{i n}\left(k^{\prime}\right) f\left(x_{i n}\right)-I\left(k^{\prime} f\right)\right|,
\end{aligned}
$$

where

$$
M(f)=\sup _{-1 \leqslant x \leqslant 1}|f(x)| .
$$

Now let $K(x)=k(x) / v(x)$, so that $v K \in L_{p}^{(1 / \rho)}$. Then we can find a polynomial $K^{\prime}$ of degree $m$ so that

$$
\left\|v\left(K-K^{\prime}\right)\right\|_{\rho}^{(1 / \rho)}<\varepsilon .
$$

If we define $k^{\prime}(x)=v(x) K^{\prime}(x)$, we have $\left\|k-k^{\prime}\right\|_{p}^{(1 / \rho)}<\varepsilon$. Hence, by the Hölder inequality,

$$
I\left(\left|k-k^{\prime}\right|\right) \leqslant\left\|k-k^{\prime}\right\|_{p}^{(1 / \rho)}\|\rho\|_{q}<A_{1} \varepsilon .
$$

Next, we have by Theorem 5 in [5] that

$$
\sup _{n}\left\|L_{n}^{f}\right\|_{\infty q}^{(\rho)}<\infty
$$

and by the Lemma in $[5$, p. 440] that

$$
\sum_{i=1}^{n}\left|w_{i n}(k)\right| \leqslant\|k\|_{p}^{(1 / \rho)}\left\|L_{n}^{f}\right\|_{\infty q}^{(\rho)} \text {. }
$$

Hence

$$
\sum_{i=1}^{n}\left|w_{i n}\left(k-k^{\prime}\right)\right| \leqslant A_{2} \varepsilon
$$

Finally, for $n$ sufficiently large so that $m+n \leqslant c n$, we have that

$$
\begin{aligned}
w_{i n}\left(k^{\prime}\right) & =\int_{-1}^{1} k^{\prime}(x) l_{i n}(x) d x=\int_{-1}^{1} v(x) K^{\prime}(x) l_{i n}(x) d x \\
& =\sum_{j=1}^{n} w_{j n}(v) K^{\prime}\left(x_{j n}\right) l_{i n}\left(x_{j n}\right)=w_{i n}(v) K^{\prime}\left(x_{i n}\right),
\end{aligned}
$$


since $l_{i n}\left(x_{j n}\right)=\delta_{i j}$. Since $K^{\prime}(x) f(x) \in R$, it follows that

$$
\begin{aligned}
\sum_{i=1}^{n} w_{i n}\left(k^{\prime}\right) f\left(x_{i n}\right) & =\sum_{i=1}^{n} w_{i n}(v) K^{\prime}\left(x_{i n}\right) f\left(x_{i n}\right) \\
& \rightarrow \int_{-1}^{1} v(x) K^{\prime}(x) f(x) d x=I\left(k^{\prime} f\right)
\end{aligned}
$$

proving the first part of our theorem.

To prove convergence of the companion rule, it suffices to show that

$$
\sum_{i=1}^{n}\left|w_{i n}\left(k^{\prime}\right)\right| f\left(x_{i n}\right) \rightarrow I\left(\left|k^{\prime}\right| f\right)=I\left(v\left|K^{\prime}\right| f\right) .
$$

First, we have as before that $\sum_{i=1}^{n} w_{i n}(v)\left|K^{\prime}\left(x_{i n}\right)\right| f\left(x_{i n}\right) \rightarrow I\left(v\left|K^{\prime}\right| f\right)$. Second, $\left|w_{i n}\left(k^{\prime}\right)\right|=\left|w_{i n}(v)\right|\left|K^{\prime}\left(x_{i n}\right)\right|$ and third, $\sum_{i=1}^{n}\left|w_{i n}(v)\right| \rightarrow I(v)=\sum_{i=1}^{n} w_{i n}(v)$, so that for any bounded function $g$,

$$
\left|\sum_{i=1}^{n}\left(\left|w_{i n}(v)\right|-w_{i n}(v)\right) g\left(x_{i n}\right)\right| \leqslant M(g) \sum_{i=1}^{n}\left(\left|w_{i n}(v)\right|-w_{i n}(v)\right) \rightarrow 0 .
$$

Combining these concludes the proof of Theorem 1 .

REMARK. Theorem 1 has been stated in terms of the convergence of $I_{n}(f$; $k) \rightarrow I(k f)$ for spaces of $k$ and $f$. Using Theorem 5 in [5], the statement could equally well have been in terms of the equivalent Lagrangian mean convergence for continuous functions.

COROLlaRY 1. Let $w \in A$ and let the points in $X_{n}$ be the zeros of $q_{n}^{(r, s)}(x)$ given by (5), where $r, s \in\{0,1\}$. Then, if $\bar{w}(x)=w(x) / w^{(r, s)}(x) \in A, I_{n}(f ; k) \rightarrow$ $I(k f)$ for all $f \in R$ and all $k$ satisfying

$$
\int_{-1}^{1} \frac{|k(x)|^{2}}{\bar{w}(x)} d x<\infty .
$$

Similarly the companion rule converges to $I(|k| f)$.

Proof. Set $p=q=2, v=\bar{w}$ and $\rho=\bar{w}^{1 / 2}$ in Theorem 1. Then the rules $I_{n}(f ; \bar{w})$ are Gauss, Radau or Lobatto rules depending on the values of $r$ and $s$ and are exact for all polynomials of degree $\leqslant 2 n-3$. In addition, all the weights $w_{i n}(\bar{w})>0, \rho \in L_{2}$ and $k \in L_{2}^{(1 / \rho)}$. Since $I_{n}(f ; k) \rightarrow I(k f)$ for all $f \in C$ and all $k \in L_{2}^{(1 / \rho)}$, the results follow. The convergence of $I_{n}(f ; k) \rightarrow I(k f)$ for all $f \in C$ is shown in [4] for $r=s=0$ and in [6] for $r=s=1$. The proof for the case $r+s=1$ is similar to, and even simpler than, the proof in [6].

For $r \doteq s=0$, this result was proved in [5]. For the other cases, the result is new. 
Corollary 2. Let $w \in G S J$ be given by (6), let $r, s \in\{0,1\}$ and let the points of $X_{n}$ be the zeros of $q_{n}^{(r, s)}(x)$ given by (5). Assume that $k$ and $w$ satisfy the following conditions:

$$
\begin{gathered}
\bar{w} \equiv w / w^{(r, s)} \in L_{1}, k \in L_{p} \\
(1-x)^{-r+1 / 4}(1+x)^{-s+1 / 4} w^{1 / 2} \in L_{1} ; \\
k(x)(1-x)^{r-1 / 4}(1+x)^{s-1 / 4} w^{-1 / 2} \in L_{p} \text { for some } p>1 .
\end{gathered}
$$

Then, for all $f \in R, I_{n}(f ; k) \rightarrow I(k f)$ and the companion rule $\rightarrow I(|k| f)$.

Proof. Set $p>1$ and

$$
\rho(x)=(1-x)^{A}(1+x)^{B} \prod_{j=1}^{m}\left|x-t_{j}\right|^{C_{j}}
$$

in Theorem 1, where

$$
\begin{aligned}
A & =\max [(2 \alpha+1-4 r) / 4,0], \\
B & =\max [(2 \beta+1-4 s) / 4,0], \\
C_{j} & =\max \left[\gamma_{j} / 2,0\right], j=1, \ldots, m .
\end{aligned}
$$

Then $\rho \in L_{q}$. Furthermore $\bar{w} \in A$ and the rule $I_{n}(f ; \bar{w})$ based on $X_{n}$ is a Gauss, Radau or Lobatto rule depending on $r$ and $s$. Since the conditions on $\rho, w$ and $k$ are such that $I_{n}(f ; k) \rightarrow I(k f)$ for all $f \in C[2]$, it follows as in the proof of Corollary 1 that the above holds for all $f \in R$ and for the companion rule.

This corollary yields the same result as Theorem $1^{\prime}$ in [3] in a much simpler fashion. However, it does not cover the case when $\bar{w}$ is not admissible. For some such $w$, we still have convergence for all $f \in C[2]$. In the next theorem, we shall prove the same for all $f \in R$ as well as for the companion rule.

ThEOREM 2. Let all the conditions of Corollary 2 hold except for the condition that $\bar{w} \in L_{1}$. Then the conclusions of Corollary 2 hold.

Proof. As in [3], we shall prove convergence for the modified rule

$$
\hat{I}_{n}(f ; k)=\sum_{i=1+r}^{n-s} w_{i n}(k) f\left(X_{i n}\right) \text {, }
$$

since it follows from the convergence of $I_{n}(f ; k)$ for $f \in C$, that $w_{1 n}(k)$, when $r=1$, and $w_{n n}(k)$, when $s=1, \rightarrow 0$. 
Proceeding as in the proof of Theorem 1, we have

$$
\begin{aligned}
\left|I(k f)-\hat{I}_{n}(f ; k)\right| \leqslant & M(f)\left[I\left(\left|k-k^{\prime}\right|\right)+\left|\sum_{i=1+r}^{n-s} w_{i n}\left(k-k^{\prime}\right)\right|\right] \\
& +\left|\sum_{i=1-r}^{n-s} w_{i n}\left(k^{\prime}\right) f\left(x_{i n}\right)-I\left(f ; k^{\prime}\right)\right| .
\end{aligned}
$$

Setting $K(x)=w^{(r, s)}(x) k(x) / w(x)$, we have that $w K \in L_{p}^{(0)}$ where $\sigma=$ $1 /\left(w^{(r, s)} \rho\right)$, and $\rho$ is given by (7). Hence we can find a polynomial $K^{\prime}$ of degree $m$ such that $\left\|w\left(K-K^{\prime}\right)\right\|_{p}^{(0)}<\varepsilon$ so that if $k^{\prime}(x)=w(x) K^{\prime}(x) / w^{(r, s)}(x)$, then $\left\|k-k^{\prime}\right\|_{p}^{(1 / p)}<\varepsilon$. Hence, as before, the first two terms on the right hand side of (8) are small. For the third term, we shall show that

$$
w_{i n}\left(k^{\prime}\right)=w_{i n}(w) K^{\prime}\left(x_{i n}\right) / w^{(r, s)}\left(x_{i n}\right), \quad 1+r \leqslant i \leqslant n-s .
$$

Then it will follow that

$$
\begin{aligned}
\sum_{i=1+r}^{n-s} w_{i n}\left(k^{\prime}\right) f\left(x_{i n}\right) & =\sum_{i=1+r}^{n-s} \frac{w_{i n}(w) K^{\prime}\left(x_{i n}\right) f\left(x_{i n}\right)}{w^{(r, s)}\left(x_{i n}\right)} \\
& \rightarrow \int_{-1}^{1} \frac{w(x) K^{\prime}(x) f(x)}{w^{(r, s)}(x)} d x=I\left(k^{\prime} f\right) .
\end{aligned}
$$

This last result is true since the second term is a Gaussian integration rule with respect to $w$ applied to the function $K^{\prime}(x) f(x) / w^{(r, s)}(x)$. Now, since the integral exists for all $f \in R$ and $w / w^{(r, s)} \notin L_{1}$, it follows that $K^{\prime}(x)$ has a zero at $x=1$ and/or at $x=-1$. Hence $K^{\prime} f / w^{(r, s)} \in R$ and we have convergence. This covers the cases $r+s=1$. When $r=s=1$, it may be that $w(x) /(1-x)$ or $w(x) /(1$ $+x)$ is in $L_{1}$. In this case, say $w(x) /(1-x) \in L_{1}, w_{i n}(w) /\left(1-x_{i n}\right)$ is a Radau weight and $K^{\prime}(x)$ has a zero at $x=-1$, so that $w K^{\prime} f /(1+x) \in R$ and again (10) holds.

Finally, to show (9), we write

$$
\begin{aligned}
w_{i n}\left(k^{\prime}\right) & =\int_{-1}^{1} k^{\prime}(x) q_{n}^{(r, s)}(x) d x /\left\{\left(x-x_{i n}\right)\left[q_{n}^{(r, s)}(x)\right]_{x=x_{i n}}^{\prime}\right\} \\
& =\int_{-1}^{1} \frac{w(x) K^{\prime}(x)}{w^{(r, s)}(x)} \frac{w^{(r, s)}(x) p_{n-r-s}(x ; w)}{\left(x-x_{i n}\right) w^{(r, s)}\left(x_{i n}\right) p_{n-r-s}^{\prime}\left(x_{i n} ; w\right)} d x \\
& =\int_{-1}^{1} \frac{w(x) K^{\prime}(x)}{w^{(r, s)}\left(x_{i n}\right)} \hat{l}_{i n}(x) d x \\
& =\frac{1}{w^{(r, s)}\left(x_{i n}\right)} \sum_{j=1}^{n} w_{j n}(w) K^{\prime}\left(x_{j n}\right) \hat{l}_{i n}\left(x_{j n}\right) \\
& =w_{i n}(w) K^{\prime}\left(x_{i n}\right) / w^{(r, s)}\left(x_{i n}\right),
\end{aligned}
$$

where $\hat{l}_{i n}(x)=p_{n-r-s}(x ; w) /\left(x-x_{i n}\right) p_{n-r-s}^{\prime}\left(x_{i n} ; w\right)$ and $\hat{l}_{i n}\left(x_{j n}\right)=\delta_{i j}$. This completes the proof of Theorem 2. 


\section{References}

[1] P. J. Davis and P. Rabinowitz, Methods of numerical integration, 2nd Edition (Academic Press, New York, 1984).

[2] P. Rabinowitz, "The convergence of interpolatory product integration rules". BIT 26 (1986), 131-134.

[3] P. Rabinowitz, "On the convergence of interpolatory product integration rules based on Gauss, Radau and Lobatto points", Israel J. Math. 56 (1986) 66-74.

[4] I. H. Sloan, "On the numerical evaluation of singular integrals". BIT 18 (1978) 91-102.

[5] I. H. Sloan and W. E. Smith, "Properties of interpolatory product integration rules", SIAM J. Numer. Anal. 19 (1982) 427-442.

[6] W. E. Smith, "Erdős-Turán mean convergence for Lagrange interpolation at Lobatto points", Bull. Austral. Math. Soc. 34 (1986) 375-381. 